\title{
BEDT-TTF organic superconductors: the entangled role of phonons
}

\author{
Alberto Girlando, ${ }^{1}$ Matteo Masino, ${ }^{1}$ Aldo Brillante, ${ }^{2}$ Raffaele G. Della Valle, ${ }^{2}$ and Elisabetta Venuti ${ }^{2}$ \\ ${ }^{1}$ Dip. Chimica Gen.Inorg. Chim.Anal. e Chim.Fis. and INSTM-UdR Parma, Parma University, 43100 Parma, Italy \\ ${ }^{2}$ Dip. Chimica Fisica ed Inorganica and INSTM-UdR Bologna, Bologna University, Bologna, Italy
}

(Dated: November 3, 2018)

\begin{abstract}
We calculate the lattice phonons and the electron-phonon coupling of the organic superconductor $\kappa$-(BEDT-TTF $)_{2} \mathrm{I}_{3}$, reproducing all available experimental data connected to phonon dynamics. Low frequency intra-molecular vibrations are strongly mixed to lattice phonons. Both acoustic and optical phonons are appreciably coupled to electrons through the modulation of the hopping integrals (e-LP coupling). By comparing the results relevant to superconducting $\kappa$ - and $\beta^{*}-(\mathrm{BEDT}-\mathrm{TTF})_{2} \mathrm{I}_{3}$, we show that electron-phonon coupling is fundamental to the pairing mechanism. Both e-LP and electron-molecular vibration $(e-\mathrm{MV})$ couplings are essential to reproduce the critical temperatures. The $e$-LP coupling is stronger, but $e-\mathrm{MV}$ is instrumental to increase the average phonon frequency.
\end{abstract}

Organic superconductors (oSC) have been studied for more than twenty years, but the nature of the pairing mechanism is still unknown, and remains one of the most intriguing problems in this class of materials [1]. Like high $T_{c}$ superconductors, oSC are low-dimensional systems characterized by strong electronic correlations. This fact and the proximity of the superconducting phase to magnetically or charge ordered states has brought about the suggestion of exotic pairing, mediated for instance by spin or charge fluctuations [2]. However, direct experimental evidence for such kind of purely electronic mechanisms has not been provided. On the other hand, the oSC phonon structure is very complex, preventing a clear assessment of the role of phonons in the pairing. Indeed, scattered experimental evidences of the involvement of intra-molecular phonons [3], of optical intermolecular phonons [4], and of acoustic phonons [5] have been presented. However, all these studies, focusing on a particular type of phonon, failed to offer a comprehensive and convincing picture of the phonon mediated mechanism in oSC. Aim of this letter is to provide a unified framework for the description of the phonon dynamics and of the electron-phonon coupling in oSC based on bisethylendithio-tetrathiafulvalene (BEDT-TTF). We shall show that all the above types of phonons are coupled to electrons, and cooperate in establishing electron pairing.

In molecular crystals it is convenient to start by distinguishing between intra- and inter-molecular vibrational degrees of freedom, and to consider their interactions at a later stage. Thus, in the framework of a tight-binding description of the electrons, intra-molecular vibrations modulate the on-site energy and yield the electron-molecular vibration ( $e-\mathrm{MV})$ coupling, while lattice phonons, mainly inter-molecular in character, modulate the hopping integrals ( $e$-LP coupling).

Molecular vibrations and $e$-MV coupling constants are transferable among different crystalline phases, and either computational or experimental methods can be used for their determination. Indeed, BEDT-TTF molecular normal modes and $e$-MV coupling constants are fairly well known [6]. The problem of lattice phonons is much harder to deal with, because the experimental characterization of lattice phonons and of $e$-LP coupling constants is difficult and subject to considerable experimental uncertainty [4. Moreover, the phonon properties are not transferable among different crystals. To overcome these difficulties, we have adopted the "Quasi Harmonic Lattice Dynamics" (QHLD) computational method [8, 8, which is known to accurately predict the crystal structure and the phonon dynamics of complex molecular crystals as a function of pressure $p$, and temperature $T$.

As extensively discussed elsewhere [7, 8], in QHLD the vibrational contribution to the crystal Gibbs energy $G(p, T)$ is approximated by the free energy of the phonons calculated in the harmonic approximation. The structure at a given $p, T$ is determined self-consistently by minimizing $G(p, T)$ with respect to lattice parameters, molecular positions and orientations. The method relies on an accurate inter-molecular potential model, which we have described as the sum of empirical atom-atom potentials, known to be reasonably transferable among crystals containing similar molecules. We have restricted our attention to the (BEDT-TTF $)_{2} \mathrm{I}_{3}$ salts, which constitute an ideal benchmark for our approach. Our strategy has been to determine and to fix once and for all the needed C, S, H and I atom-atom parameters on the basis of the crystal structures of neutral BEDT-TTF and nonsuperconducting $\alpha$-(BEDT-TTF $)_{2} \mathrm{I}_{3}$ salt [8]. We have then applied the method to the phonon structure and $e$-LP coupling strength of superconducting $\beta^{*}$-(BEDT$\mathrm{TTF})_{2} \mathrm{I}_{3}$ salt [7]. Here we illustrate the corresponding results for $\kappa$-(BEDT-TTF $)_{2} \mathrm{I}_{3}$. The comparison with the experimental data for the two superconducting $\beta^{*}$ - and $\kappa$-phases allows us to validate our approach and to assess the role of phonons in the superconductivity mechanism.

Since both $\mathrm{I}_{3}^{-}$and BEDT-TTF present low frequency intra-molecular modes falling in the region of the lattice phonons, the coupling between inter- and intra-molecular phonons may be important for the (BEDT-TTF $)_{2} \mathrm{I}_{3}$ salts. To account for this effect, we use the exciton-like model described elsewhere [7]. We stress that lattice phonons are actually mixed only with low-frequency vibrations 


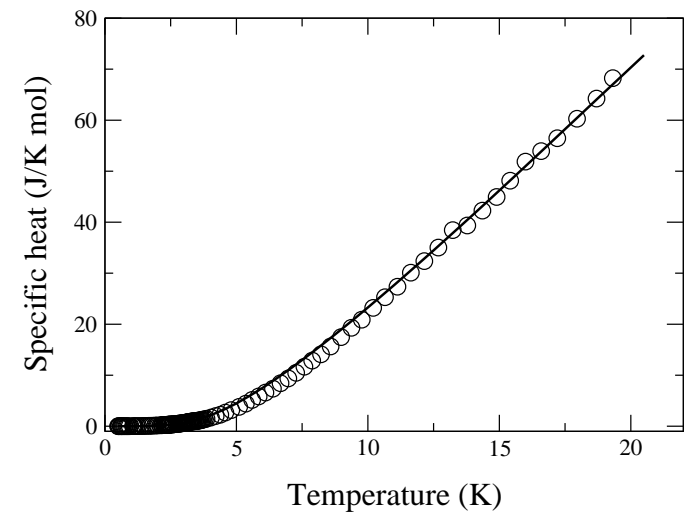

FIG. 1: Calculated ( $c_{V}$, full line) and experimental $\left(c_{p}\right.$, circles, from Ref. 10) $\kappa$-(BEDT-TTF $)_{2} \mathrm{I}_{3}$ specific heat.

(below $300 \mathrm{~cm}^{-1}$ ), and these do not modulate on-site energies [6]. Therefore $e$-LP and $e$-MV couplings can be dealt with separately.

At room temperature, $\kappa$-(BEDT-TTF $)_{2} \mathrm{I}_{3}$ crystallizes in the monoclinic system $P 2_{1} / c$, with four molecules per unit cell. Upon cooling below $150 \mathrm{~K}$ the crystal structure changes to the $P 2_{1}$ system [9]. The BEDT-TTF layer is in the $b c$ plane. The QHLD calculations reproduce both crystal structures, which correspond to two distinct $G(p, T)$ minima. The calculated lattice parameters are within $3 \%$ of the experimental ones. The results presented below all refer to the low temperature $\kappa$-phase.

A comparison with $\kappa$-(BEDT-TTF $)_{2} \mathrm{I}_{3}$ scanty infrared and Raman data does not offer a stringent test for the calculated phonon frequencies, whose number largely exceeds that of the currently observed bands. We then compare the experimental [10] specific heat $c_{p}$ to the calculated $c_{V}$, which depends on the overall distribution of phonon frequencies $\omega_{\mathbf{q} j}: c_{V}(T)=k_{\mathrm{B}} \sum_{\mathbf{q} j} F\left(\hbar \omega_{\mathbf{q} j} / k_{\mathrm{B}} T\right)$, where $F(x)=x^{2} \mathrm{e}^{x}\left(\mathrm{e}^{x}-1\right)^{-2}$ and the sum is extended to all phonon branches $j$ and wavevectors q. As shown in Fig. 1, the agreement is outstanding. We stress that there are no adjustable parameters in our $c_{V}(T)$ calculation.

To calculate the $e$-LP coupling strength we assume that the low frequency phonons, mainly intermolecular in character, couple to electrons only through the modulation of hopping integrals:

$$
t_{K L}=t_{K L}^{0}+\sum_{j} g(K L ; \mathbf{q} j) Q_{\mathbf{q} j}
$$

where $t_{K L}$ is the hopping integral between neighboring pairs $K L$ of BEDT-TTF molecules, and $Q_{\mathbf{q} j}$ are the dimensionless normal coordinates. Finally, $g(K L ; \mathbf{q} j)=$ $\sqrt{\hbar / 2 \omega_{\mathbf{q} j}}\left(\partial t_{K L} / \partial Q_{\mathbf{q} j}\right)$ defines the linear $e$-LP coupling constants. The $t_{K L}^{0}$ and their dependence from the QHLD eigenvectors $Q_{\mathbf{q} j}$ have been calculated by the extended Hückel (EH) method [7]. The obtained $t^{0}$ values are in good agreement with those calculated by extended basis set density functional methods [1]. The $t^{0}$ are

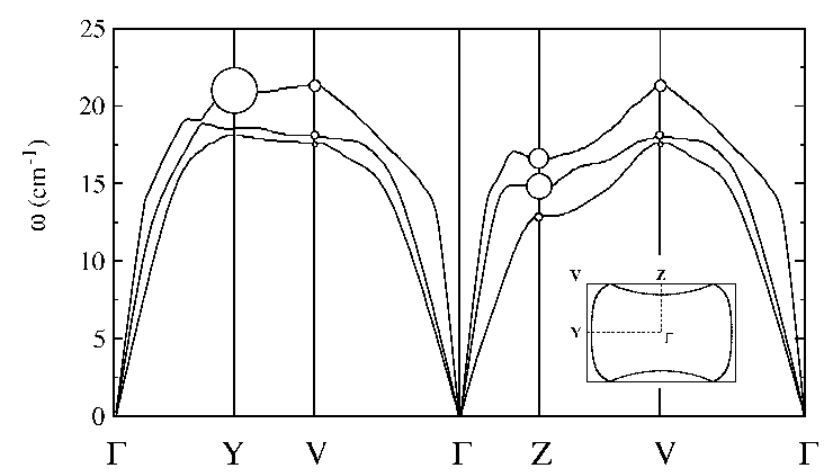

FIG. 2: Dispersion curves of $\kappa$-(BEDT-TTF $)_{2} \mathrm{I}_{3}$ acoustic lattice phonons along the principal directions shown in the inset. Inset: Fermi surface calculated with the dimer model.

used to describe the band structure in a tight binding approach, adopting the dimer model [12]. The resulting conduction bands and Fermi surface (inset of Fig. 2) match closely those obtained considering all the four site orbitals within the unit cell [9].

To express the $e$-LP coupling constants in reciprocal space, we have to introduce the dependence on both electronic (k) and phononic (q) wavevectors. We have followed the approximation scheme adopted for $\beta^{*}$-(BEDT$\mathrm{TTF})_{2} \mathrm{I}_{3}[$ []. The $\mathbf{k}$ dependence has been accounted for in terms of the dimer model: the coupling constants associated with the modulation of the intra-dimer hopping integral are assumed independent of $\mathbf{k}$, whereas the inter-dimer g's are assumed to have the following dependence: $\mathrm{g}\left(\mathbf{k}, \mathbf{k}^{\prime} ; j\right)=\sum_{\mathbf{R}} g_{K L}(\mathbf{q} j)\left(\mathrm{e}^{i \mathbf{k}^{\prime} \mathbf{R}}-\mathrm{e}^{i \mathbf{k} \mathbf{R}}\right)$, where $\mathbf{q}=\mathbf{k}^{\prime}-\mathbf{k}$, and $\mathbf{R}$ represents the nearest neighbor lattice vectors. For what concerns the $\mathbf{q}$ dependence, the optical modes are taken independent of $\mathbf{q}$, whereas for acoustic phonons we have simply assumed $g_{K L}(\mathbf{q} j) \propto \sqrt{|\mathbf{q}|}$ up to the average value calculated at the zone edges.

Fig. 2 shows the frequency dispersion of the acoustic modes along representative Brillouin zone directions, together with a pictorial representation of the coupling strength at the zone edges. The dots diameters are proportional to the squared coupling constant, which are much larger along the $\mathrm{Y}, \mathrm{Z}$ directions (i.e. the $b^{*}, c^{*}$ axes) than along the diagonal. Our results indicate that for $\kappa$-(BEDT-TTF $)_{2} \mathrm{I}_{3}$ the coupling of acoustic phonons is very anisotropic.

From the coupling constants in reciprocal space and the QHLD phonon density of states we calculate the Eliashberg coupling function [13]: $\alpha^{2}(\omega) F(\omega)=$ $N\left(E_{F}\right) \sum_{j}\left\langle\left|\mathrm{~g}\left(\mathbf{k}, \mathbf{k}^{\prime} ; j\right)\right|^{2} \delta\left(\omega-\omega_{\mathbf{q} j}\right)\right\rangle_{F S}$, where $N\left(E_{F}\right)$ is the unit cell density of states/spin at the Fermi level, and \langle\rangle$_{F S}$ indicate the average over the Fermi surface. Fig. 3 compares $\kappa$ - and $\beta^{*}$-(BEDT-TTF $)_{2} \mathrm{I}_{3}$ Eliashberg functions. Both curves have been smoothed by a Gaussian of constant width to ease the comparison. Although significant differences exist, also due to the higher number of phonons for the $\kappa$-phase unit cell, the most significant 


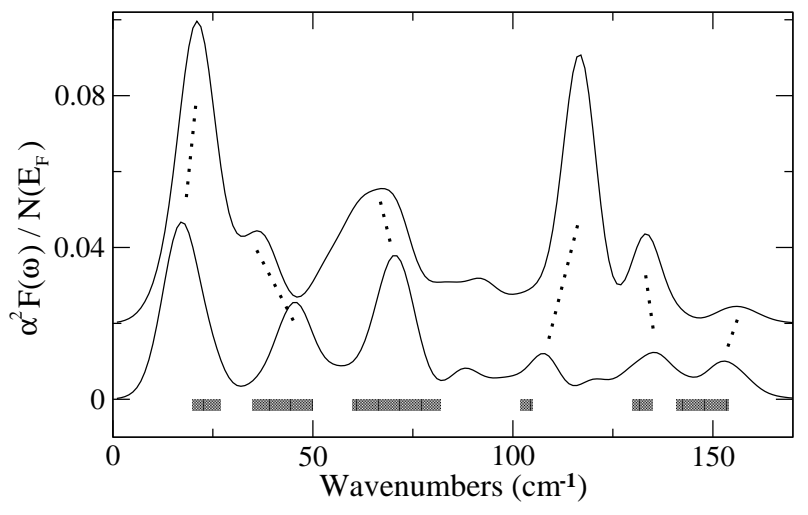

FIG. 3: Calculated Eliashberg function of $\kappa$-(BEDT-TTF $)_{2} \mathrm{I}_{3}$ (lower curve) and $\beta^{*}$-(BEDT-TTF) ${ }_{2} \mathrm{I}_{3}$ (upper curve, offset for clarity). The shaded areas at the bottom indicate the position of the most strongly coupled phonons, as detected from Raman on others $\kappa$-phase BEDT-TTF salts (from Ref. 4).

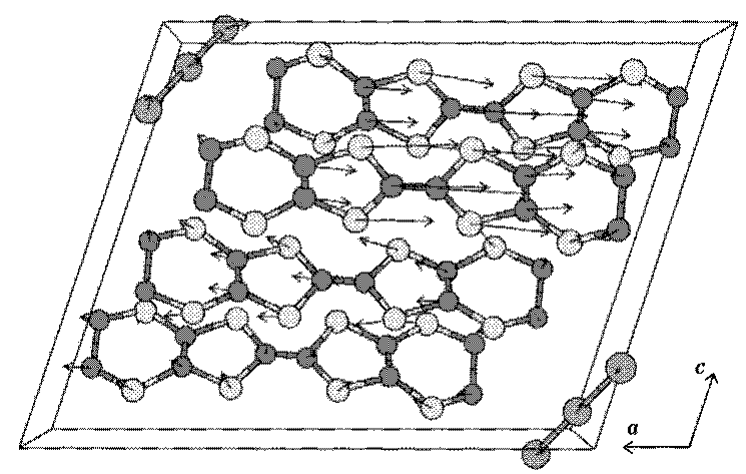

FIG. 4: Eigenvector of $\kappa$-(BEDT-TTF $)_{2} \mathrm{I}_{3}$ zone edge $(\mathrm{Y})$ acoustic phonon at $21 \mathrm{~cm}^{-1}$.

peaks are correlated, as indicated by the dotted lines. In both salts the lowest frequency peak results from the overlap of acoustic and lowest frequency optical modes.

Comparison of the computed Eliashberg function with available experimental data provides a test for the $e$-LP constants and QHLD eigenvectors. For the $\beta$ phase, the calculation compares quite favorably [7] with the Eliashberg function directly derived from point contact measurements. This kind of measurement is not available for $\kappa$-(BEDT-TTF $)_{2} \mathrm{I}_{3}$, but we can make reference to the phonon self-energy effects measured by Raman on $\kappa$-(BEDT-TTF $)_{2} \mathrm{Cu}(\mathrm{NCS})_{2}$ and $\kappa$-(BEDT$\mathrm{TTF})_{2} \mathrm{Cu}\left[\mathrm{N}(\mathrm{CN})_{2}\right] \mathrm{Br}$ [4]. A quantitative comparison is not possible, due to the different counter-ions. Nevertheless, Fig. 3 shows that the frequency regions (shaded areas at the bottom) of the few strongly coupled optical phonons 胞, indeed correspond to the peaks of $\kappa$-(BEDT$\mathrm{TTF})_{2} \mathrm{I}_{3}$ Eliahsberg function. We finally mention that inelastic neutron scattering on $\kappa$-(BEDT-TTF $)_{2} \mathrm{Cu}(\mathrm{NCS})_{2}$ [5] evidences also the involvement of acoustic phonons, in agreement with our findings.
Although further measurements, like Raman spectra with excitation in the infrared, are highly desirable to provide further tests, we believe that the above comparison already gives confidence to the QHLD eigenvectors, and as an example we show in Fig. A the eigenvector corresponding to $\kappa$-(BEDT-TTF $)_{2} \mathrm{I}_{3}$ zone edge acoustic phonon at $21 \mathrm{~cm}^{-1}$. As it occurs in several other cases, the coupling is induced by the relative displacement of BEDT-TTF along the long molecular axis, i.e. roughly normal to the BEDT-TTF layers. Moreover, a normal mode coupled to electron is in general a mixture of both BEDT-TTF and $\mathrm{I}_{3}$ vibrations. This mode mixing indicates that also the counter-ion vibrations may play an indirect role in the coupling.

We now proceed to estimate the critical temperatures. From Fig. 3 Eliashberg function we derive the dimensionless coupling constant $\lambda^{\mathrm{LP}}$, and the logarithmic average frequency $\omega_{\mathrm{ln}}^{\mathrm{LP}}[13$ :

$$
\begin{gathered}
\lambda^{\mathrm{LP}}=2 \int_{0}^{\omega_{\max }} \frac{\alpha^{2}(\omega) F(\omega)}{\omega} d \omega \\
\omega_{\ln }^{\mathrm{LP}}=\exp \left(\frac{2}{\lambda_{\mathrm{LP}}} \int_{0}^{\omega_{\max }} \frac{\alpha^{2}(\omega) F(\omega)}{\omega} \ln \omega d \omega\right)
\end{gathered}
$$

The $\lambda$ value depends on $N\left(E_{F}\right)$. With our tight binding EH calculation we estimate $N\left(E_{F}\right)=5.4$ and 3.8 spin states/(eV unit cell), so that $\lambda^{\mathrm{LP}}=0.59$ and 0.65 for $\kappa$ and $\beta^{*}$-(BEDT-TTF $)_{2} \mathrm{I}_{3}$, respectively. The corresponding $\omega_{\mathrm{ln}}^{\mathrm{LP}}$ values are 27 and $40 \mathrm{~cm}^{-1}$. When these values are inserted into the Allen-McMillan equation [13]:

$$
T_{c}=\frac{\omega_{\ln }}{1.2} \exp \left[-\frac{1.04(1+\lambda)}{\lambda-\mu^{*}(1+0.62 \lambda)}\right],
$$

assuming the standard 0.1 value for the Coulomb pseudopotential $\mu^{*}$, one obtains $T_{c}=0.8$ and $1.7 \mathrm{~K}$ for $\kappa$ and $\beta^{*}$-(BEDT-TTF $)_{2} \mathrm{I}_{3}$, respectively, to be compared with the experimental values of 3.4 and $8.1 \mathrm{~K}$, 10 . Of course, the $T_{c}$ values depends critically on $N\left(E_{F}\right)$, a parameter difficult to evaluate 14. However, even by taking the maximum current estimates, derived from experiment and therefore enhanced by many body electronelectron and electron-phonon effects [14], the $T_{c}$ values $(2.7$ and $3.6 \mathrm{~K})$ remain significantly below the experiment. Therefore $e$-LP coupling alone cannot reasonably account for the $T_{c}$ of the two salts.

However, one must not forget high frequency $e-\mathrm{MV}$ coupled phonons [6, 15]. By including these phonons into the calculation of $\lambda$ and $\omega_{\ln }$, the above scenario changes substantially. We underline that $e-\mathrm{MV}$ coupling gives an additive contribution to the total $\lambda, \lambda=\lambda^{\mathrm{LP}}+\lambda^{\mathrm{MV}}$, whereas the contribution to $\omega_{\ln }$ is multiplicative: $\omega_{\ln }=$ $\omega_{\ln }^{\mathrm{LP}} \omega_{\ln }^{\mathrm{MV}}$, where $\omega_{\ln }^{\mathrm{MV}}=\exp \left[\frac{2 N\left(E_{F}\right)}{N \lambda} \sum_{l} \frac{g_{l}^{2}}{\omega_{l}} \ln \omega_{l}\right]$, with $g_{l}$ the $e-\mathrm{MV}$ coupling constants and $N$ the number of molecules per unit cell 15. It turns out that by including the $e$-MV coupling, $\lambda$ increases by only $\sim 25 \%$, but $\omega_{\mathrm{ln}}$ reaches 60 and $94 \mathrm{~cm}^{-1}$ for $\kappa$ - and $\beta^{*}$-(BEDT$\mathrm{TTF})_{2} \mathrm{I}_{3}$, respectively. By using these new values into 
Eq. (3), keeping $\mu^{*}=0.1$, we find that our tight binding EH $N\left(E_{F}\right)$ estimates, which are within the range of the currently accepted values [14, account for the observed critical temperatures. In particular, precise $T_{c}$ matching requires $N\left(E_{F}\right)=5.2$ and 3.9 spin states $/(\mathrm{eV}$ unit cell), corresponding to a total $\lambda$ of 0.74 and 0.91 for $\kappa$ - and $\beta^{*}$-(BEDT-TTF $)_{2} \mathrm{I}_{3}$, respectively. We finally remark that only by using the new $\omega_{\mathrm{ln}}$, which does not depend on $N\left(E_{F}\right)$, the empirical relation [16] connecting this parameter to the specific heat jump $\Delta C_{p} / \gamma T_{c}$ yields for $\kappa$-(BEDT-TTF $)_{2} \mathrm{I}_{3}$ a jump in nice agreement with experiment (1.7 vs 1.6) [10].

In our approach electron-phonon coupling provides a consistent and plausible explanation of many experimental findings related to the superconducting properties of $\kappa$ - and $\beta^{*}$-phase BEDT-TTF salts. Besides the just mentioned $T_{c}$ values and specific heat jump, the scattered experimental evidences of the involvement of acoustic and optical lattice phonons have been rationalized. The coupling of acoustic phonons in the $\kappa$-phase turns out to be strongly anisotropic, likely yielding an anisotropic gap that might confuse the node search [17. In addition, we have found that the most strongly $e$-LP coupled BEDTTTF modes imply motions approximately normal to the conducting planes, with counter-ions vibrations mixed to BEDT-TTF ones (cf. Fig. (1). This finding might well account for the "interlayer effects" detected in thermal expansion measurements [18]. Experimental evidences in favor of the involvement of $e-\mathrm{MV}$ coupled phonons are mainly related to isotopic effects [3, 19]. In particular, the universal inverse deuterium isotope effects $(0.25 \mathrm{~K})$ is likely due to a small increase in $\omega_{\mathrm{ln}}$. The $e$-MV coupling constants of deuterated BEDT-TTF are not known, but we have verified that very small and plausible changes of the coupling constants with respect to those of pristine BEDT-TTF [6] may indeed lead to the required increase in $T_{c}$, despite the large downwards shift of several frequencies upon deuteration.

The principal conclusion of our paper is that phonons are the main responsible for the coupling mechanism in BEDT-TTF based oSC. The role of phonons is complex, or "entangled", in the sense that different kinds of phonons are all important, but contribute in different ways. The acoustic and optical lattice phonons, modulating $t$, give the main weight to $\lambda$, but intra-molecular phonons modulating on-site energies are essential in increasing the average $\omega_{\ln }$ value. Although here we vindicate the role of phonons, we also stress that traditional phonon mediated pairing alone cannot give reason of all the experimental findings related to superconducting properties of BEDT-TTF salts. The proximity of antiferromagnetic or charge ordered states in many oSC clearly indicate that electron-electron interactions are important [1], and these cannot be properly accounted for in our effective single particle approach. In particular, just con- sider the above mentioned $\kappa$-(BEDT-TTF $)_{2} \mathrm{Cu}(\mathrm{NCS})_{2}$ and $\kappa$-(BEDT-TTF $)_{2} \mathrm{Cu}\left[\mathrm{N}(\mathrm{CN})_{2}\right] \mathrm{Br}$ salts. Their $\omega_{\ln }$ is similar to $\kappa$-(BEDT-TTF $)_{2} \mathrm{I}_{3}$, yet their $T_{c}$ 's are 9.5 and $11.5 \mathrm{~K}$, respectively [20]. We think it is unlikely that the corresponding increase in $\lambda$ with respect to $\kappa$-(BEDT$\mathrm{TTF})_{2} \mathrm{I}_{3}$ can be attributed to a significant change in the $e$-LP coupling strength due to the change in counterions. Rather, we believe that electron-electron interactions and/or the proximity of a phase boundary may lead to an enhancement of the effective electron-phonon coupling. In other words, we need to analyze the role of electron-phonon coupling in low-dimensional strongly correlated materials, still a poorly understood topic [21. Our work represents the necessary prerequisite for such an analysis in BEDT-TTF-based oSC.

Work supported by the Italian Ministero Istruzione, Università e Ricerca (M.I.U.R.). We thank L. Frediani for the DFT calculations, and A. Painelli for discussions.

[1] J. Wosnitza, Curr. Op. Solid State Mater. Science, 5, 131 (2001); J. Singleton, Rep. Progr. Phys. 63, 1111 (2000).

[2] J. Schmalian, Phys. Rev. Lett. 81, 4232 (1998); J. Merino and R.H. McKenzie, Phys. Rev. Lett. 87, 237002 (2001).

[3] V. Merzhanov et al., C.R. Acad. Sci. Paris, 314, 563 (1992).

[4] D. Pedron et al., Physica C 276, 1 (1998); E. Falques et al., Phys. Rev. B 62, R9291 (2000); D. Pedronet al., Synth. Metals 103, 2220 (1999).

[5] L. Pintschovious et al., Europhys. Lett. 37, 627 (1997).

[6] G. Visentini et al., Phys. Rev. B 58, 9460 (1998), and references therein.

[7] A. Girlando et al., Phys. Rev. B 62, 14476 (2000).

[8] A. Brillante et al., Chem. Phys. Lett. 274, 478 (1997); R.G. Della Valle et al., Physica B, 265, 195 (1999).

[9] H. Kobayashi et al., J. Mater. Chem. 5, 1681 (1995).

[10] J. Wosnitza et al., Phys. Rev. B 50, 12747 (1994).

[11] The $t$ values are available on request. See also A. Fortunelli and A. Painelli, Phys. Rev. B 55, 16088 (1997).

[12] G. Visentini et al., Europhys. Lett. 42, 467 (1998).

[13] P.B. Allen and R.C. Dynes, Phys. Rev.B 12, 905 (1975).

[14] J. Merino and R. McKenzie, Phys. Rev. B 62, 2416 (2000) and references therein.

[15] D. Pedron et al., Mol. Cryst. Liq. Cryst. 234, 161 (1993).

[16] F. Marsiglio and J.P. Carbotte, Phys. Rev. B 33, 6141 (1986).

[17] There is no agreement yet about the gap symmetry. The following studies on $\kappa$-(BEDT-TTF $)_{2} \mathrm{Cu}(\mathrm{NCS})_{2}$ are in favor of $s, d_{x^{2}-y^{2}}$ and $d_{x y}$ symmetry, respectively: J. Müller et al., Phys. Rev. B 65, 140509 (2002); J.M.Schrama et al., Phys. Rev. Lett. 88, 3041 (1999); T. Arai et al., Phys. Rev. B 63, 104518 (2001) and K.Izawa et al., Phys. Rev. Lett. 88, 027002 (2002). See also: G. Varelogiannis, Phys. Rev. Lett. 88, 117005 (2002).

[18] J. Müller et al., Phys. Rev. B 65, 144521 (2002).

[19] J.A. Schlueter et al., Physica C 351, 261 (2001).

[20] H. Elsinger et al., Phys. Rev. Lett. 84, 6098 (2000).

[21] A. Lanzara et al., Nature 412, 510 (2001). 